\title{
Iteratively Learning Electromyography (EMG)-based Functional Electrical Stimulation (FES) for Stroke Rehabilitation
}

\author{
O. Lupu ${ }^{1}$, M. Madaschi², T. Seel ${ }^{1}$, A. Cologni ${ }^{2}$, F. Previdi' ${ }^{2}$, T. Schauer ${ }^{1}$ \\ ${ }^{1}$ Technische Universität Berlin, Germany, schauer@ control.tu-berlin.de \\ ${ }^{2}$ Universita' degli Studi di Bergamo, Bergamo, Italy
}

\section{Introduction}

Rehabilitation after stroke can be improved by EMG-proportional FES. While the muscle is being stimulated proportional to its detected residual voluntary activation, the patient practices to reach pre-defined joint angles iteratively. Often the patient has bad control over the movement and oscillations occur. A potential reason is the nonlinear static EMG-angle relation resulting from EMG-proportional stimulation. This contribution describes the development of an improved EMG-based FES system that iteratively learns the nonlinear EMG-FES relation that leads to a linear relation between EMG and joint angle.

\section{Methods}

Stimulation of wrist extensor is considered. EMG is measured directly from stimulation electrodes. Starting with proportional EMG control, the subject is asked iteratively to track different patient-typical EMG levels using visual feedback. The resulting joint angles for each EMG level are measured and used to update the EMG-FES relation from iteration to iteration until the desired linear EMG-angle relation is established. In a second step the subject is asked to track a number of reference joint angles while the EMG-FES relation will be set to the nonlinear relation that the algorithm converged to.

\section{Results}

Healthy subjects were able to easily track pre-defined EMG levels. Within three iterations the system generated an almost perfectly linear EMG-FES relation. This was found to give the subject good control over his movement.

\section{Conclusion}

We developed a learning EMG-based stimulation system that establishes a linear EMG-angle relation. This was demonstrated to give good FES-supported joint angle tracking in healthy subjects. Stroke rehabilitation patients are expected to benefit from the new system. According clinical studies will be performed. 